\title{
On an extension of Nunokawa's lemma
}

\author{
Mamoru Nunokawa ${ }^{1} \cdot$ Janusz Sokół ${ }^{2}$
}

Received: 26 July 2021 / Accepted: 1 September 2021 / Published online: 15 January 2022

(c) The Author(s) 2022

\section{Abstract}

Jack's Lemma says that if $f(z)$ is regular in the disc $|z| \leq r, f(0)=0$, and $|f(z)|$ assumes its maximum at $z_{0}$ on the circle $|z|=r$, then $z_{0} f^{\prime}(z)_{0} / f\left(z_{0}\right) \geq 1$. This Lemma was generalized in several directions. In this paper we consider an improvement of some first author's results of this type.

Keywords Analytic functions - Bounded boundary rotation · Length problem · Starlike functions

Mathematics Subject Classification Primary 30C45; Secondary 30C80

\section{Introduction}

Let $\mathcal{H}$ be the family of analytic functions in the region $\mathbb{D}=\{z:|z|<1\}$ on the complex plane $\mathbb{C}$. Let $\mathcal{A} \subset \mathcal{H}$ denote the set of all functions $f(z)$ that are analytic in $\mathbb{D}$ with the series representation

$$
f(z)=z+\sum_{n=2}^{\infty} a_{n} z^{n}, \quad z \in \mathbb{D} .
$$

Also by $\mathcal{S}$ we means a subfamily of the set $\mathcal{A}$ which contains univalent functions. We next denote by $\mathcal{P}$ the class of analytic functions $p(z)$ which are normalized by

Dedicated to Sachiko Nunokawa.

$凶 \quad$ Janusz Sokół

jsokol@ur.edu.pl

Mamoru Nunokawa

mamorununokawa1983@gmail.com

1 University of Gunma, Hoshikuki-cho 798-8, Chuou-Ward, Chiba 260-0808, Japan

2 College Natural Sciences, University of Rzeszów, ul. Prof. Pigonia 1, 35-310 Rzeszow, Poland

Birkhäuser 


$$
p(z)=1+\sum_{n=1}^{\infty} p_{n} z^{n}
$$

such that $\mathfrak{R e}\{p(z)\}>0, \quad z \in \mathbb{D}$. Furthermore, by using the set $\mathcal{P}$, let $\mathcal{S}^{*}$ and $\mathcal{C}$ denote the families of starlike and convex functions in $\mathbb{D}$ which are defined as

$$
\begin{aligned}
\mathcal{S}^{*} & =\left\{f: f \in \mathcal{S} \text { and } \frac{z f^{\prime}(z)}{f(z)} \in \mathcal{P}, z \in \mathbb{D}\right\}, \\
\mathcal{C} & =\left\{f: f \in \mathcal{S} \text { and } \frac{\left(z f^{\prime}(z)\right)^{\prime}}{f^{\prime}(z)} \in \mathcal{P}, z \in \mathbb{D}\right\} .
\end{aligned}
$$

The above subfamilies of the set $\mathcal{S}$ are among the most studied families. Recall also, that if $f \in \mathcal{A}$ satisfies

$$
\mathfrak{R e}\left\{\frac{z f^{\prime}(z)}{e^{i \alpha} g(z)}\right\}>0, \quad z \in \mathbb{D}
$$

for some $g \in \mathcal{S}^{*}$ and some $\alpha \in(-\pi / 2, \pi / 2)$, then $f$ is said to be close-to-convex in $\mathbb{D}$ and denoted by $f \in \mathcal{K}$. An univalent function $f \in \mathcal{A}$ belongs to $\mathcal{K}$ if and only if the complement $E$ of the image-region $F=\{f(z):|z|<1\}$ is the union of rays that are disjoint (except that the origin of one ray may lie on another one of the rays). We note that if $g(z)=z$, then the class $\mathcal{K}$ reduces to the set $\mathcal{R}$ of bounded turning functions.

Jack's Lemma [2], says that if $f(z)$ is regular in the disc $|z| \leq r, f(0)=0$, and $|f(z)|$ assumes its maximum at $z_{0}$ on the circle $|z|=r$, then $z_{0} f^{\prime}(z)_{0} / f\left(z_{0}\right) \geq 1$. Now we will consider a lemma, which is a small extension of Jack's Lemma.

We will test behavior of $z_{0} f^{\prime}(z)_{0} / f\left(z_{0}\right)$ when $|f(z)|$ assumes its local maximum at $z_{0}$ on the following neighborhood

$$
N_{\varepsilon}\left(z_{0}\right)=\left\{z:|z| \leq\left|z_{0}\right|, \quad\left|z-z_{0}\right| \leq \varepsilon\right\} .
$$

Lemma 1.1 [1] Assume that $p(z)=a_{k} z^{k}+a_{k+1} z^{k+1}+\cdots, a_{k} \neq 0, k \geq 1$ is analytic in $\mathbb{D}$. If there exists $z_{0} \in \mathbb{D}$ such that

$$
\max _{z \in N_{\varepsilon}\left(z_{0}\right)}|p(z)|=\left|p\left(z_{0}\right)\right|
$$

for some $\varepsilon>0$, then $z_{0} p^{\prime}\left(z_{0}\right) / p\left(z_{0}\right)$ is a real number and

$$
\frac{z_{0} p^{\prime}\left(z_{0}\right)}{p\left(z_{0}\right)} \geq k \geq 0 .
$$

Proof Let us put

$$
p(z)=z^{k} \varphi(z) .
$$

Then $\varphi(z)$ is analytic in $\mathbb{D}$ and $|\varphi(z)|$ takes its local maximum value at the point $z=z_{0}$ on the circular $\operatorname{arc} z=\left|z_{0}\right| e^{i \theta}, \arg z_{0}-\varepsilon<\theta<\arg z_{0}+\varepsilon$. When $z$ moves on the circle 
$|z|=\left|z_{0}\right|$ with positive direction, then $\arg \varphi(z)$ is increasing at $z=z_{0}$. Therefore, we have

$$
\left[\frac{\mathrm{d} \arg \varphi\left(\left|z_{0}\right| e^{i \theta}\right)}{\mathrm{d}}\right]_{\theta=\theta_{0}}=\mathfrak{R e} \frac{z_{0} \varphi^{\prime}\left(z_{0}\right)}{\varphi\left(z_{0}\right)} \geq 0
$$

and so

$$
\mathfrak{R e} \frac{z_{0} p^{\prime}\left(z_{0}\right)}{p\left(z_{0}\right)}=\mathfrak{R e}\left(k+\frac{z_{0} \varphi^{\prime}\left(z_{0}\right)}{\varphi\left(z_{0}\right)}\right) \geq k \geq 0 .
$$

From the hypothesis, we have

$$
\left[\frac{\mathrm{d}\left|p\left(\left|z_{0}\right| e^{i \theta}\right)\right|}{\mathrm{d}^{\prime}}\right]_{\theta=\theta_{0}}=0
$$

so

$$
\left[\frac{\mathrm{d} \log \left|p\left(\left|z_{0}\right| e^{i \theta}\right)\right|}{\mathrm{d}}\right]_{\theta=\theta_{0}}=0
$$

too. Therefore, we have

$$
\begin{aligned}
{\left[\mathfrak{I m} \frac{\mathrm{d} \log p(z)}{\mathrm{d} \log z}\right]_{z=z_{0}} } & =\left[-\frac{\mathrm{d} \log \left|p\left(\left|z_{0}\right| e^{i \theta}\right)\right|}{\mathrm{d}}\right]_{\theta=\theta_{0}} \\
& =\left[-\left(\frac{\mathrm{d}|p(z)|}{\mathrm{d} \theta}\right) \frac{1}{|p(z)|}\right]_{\theta=\theta_{0}} \\
& =0=\mathfrak{I m} \frac{z_{0} p^{\prime}\left(z_{0}\right)}{p\left(z_{0}\right)}
\end{aligned}
$$

This shows

$$
\frac{z_{0} p^{\prime}\left(z_{0}\right)}{p\left(z_{0}\right)} \geq k \geq 0
$$

Theorem 1.2 Assume that $p(z)=1+a_{k} z^{k}+a_{k+1} z^{k+1}+\cdots, a_{k} \neq 0, k \geq 1$ is analytic in $\mathbb{D}$. Assume there exist two points $z_{1}, z_{2} \in \mathbb{D}$ such that $\left|z_{1}\right|=\left|z_{2}\right|$ and

$$
\arg p\left(z_{2}\right)=-\frac{\pi \beta}{2}, \quad \arg p\left(z_{1}\right)=\frac{\pi \alpha}{2}
$$

for some $\alpha, \beta, \alpha, \beta \in(0,2)$. If

$$
-\frac{\pi \beta}{2}<\arg p(z)<\frac{\pi \alpha}{2}, \quad|z|<\left|z_{1}\right|=\left|z_{2}\right|
$$

and

$$
\begin{aligned}
& \max _{z \in N_{\varepsilon}\left(z_{1}\right)}|\arg \{p(z)\}|=\left|\arg \left\{p\left(z_{1}\right)\right\}\right| \\
& \max _{z \in N_{\varepsilon}\left(z_{2}\right)}|\arg \{p(z)\}|=\left|\arg \left\{p\left(z_{2}\right)\right\}\right|
\end{aligned}
$$


for some $\varepsilon>0$, then we have

$$
\frac{z_{1} p^{\prime}\left(z_{1}\right)}{p\left(z_{1}\right)}=\frac{i \alpha k}{2}\left(a+\frac{1}{a}\right), \quad \frac{\alpha k}{2}\left(a+\frac{1}{a}\right) \geq \alpha k,
$$

where $p^{1 / \alpha}\left(z_{1}\right)=i a, a>0$, and

$$
\frac{z_{2} p^{\prime}\left(z_{2}\right)}{p\left(z_{2}\right)}=-\frac{i \beta k}{2}\left(b+\frac{1}{b}\right), \quad-\frac{\beta k}{2}\left(b+\frac{1}{b}\right) \leq-\beta k,
$$

where $p^{1 / \beta}\left(z_{2}\right)=-i b, b>0$.

Proof Let us check the first case $\arg p\left(z_{1}\right)=\pi \alpha / 2$ and put

$$
q(z)=[p(z)]^{1 / \alpha}, \quad \varphi(z)=\frac{1-q(z)}{1+q(z)},
$$

then it follows that

$$
q(0)=1, q^{\prime}(0)=q^{\prime \prime}(0)=\cdots=q^{(k-1)}(0)=0 \quad \text { and } \quad q^{(k)}(0) \neq 0
$$

and so

$$
\varphi(0)=\varphi^{\prime}(0)=\varphi^{\prime \prime}(0)=\cdots=\varphi^{(k-1)}(0)=0 \quad \text { and } \quad \varphi^{(k)}(0) \neq 0
$$

From the hypothesis, we have

$$
\arg q(z)<\frac{\pi}{2}, \quad z \in D_{\varepsilon}\left(z_{1}\right)=N_{\varepsilon}\left(z_{1}\right) \backslash\left\{z_{1}\right\}
$$

for some $\varepsilon>0$ and

$$
\arg q\left(z_{1}\right)=\frac{\pi}{2}
$$

Therefore, we have

$$
|\varphi(z)|<1, \quad z \in D_{\varepsilon}\left(z_{1}\right)
$$

and

$$
\left|\varphi\left(z_{1}\right)\right|=1 .
$$

This shows that $|\varphi(z)|$ takes its local maximum value at the point $z=z_{1}$ in the the domain $N_{\varepsilon}\left(z_{1}\right)$ and applying Lemma 1.1, we have

$$
\begin{aligned}
\frac{z_{1} \varphi^{\prime}\left(z_{1}\right)}{\varphi\left(z_{1}\right)} & =-\frac{z_{1} q^{\prime}\left(z_{1}\right)}{1-q\left(z_{1}\right)}-\frac{z_{1} q^{\prime}\left(z_{1}\right)}{1+q\left(z_{1}\right)} \\
& =-\frac{2 z_{1} q^{\prime}\left(z_{1}\right)}{\left(1-q\left(z_{1}\right)\right)\left(1+q\left(z_{1}\right)\right)} \\
& =-\frac{2 z_{1} q^{\prime}\left(z_{1}\right)}{(1-i a)(1+i a)}
\end{aligned}
$$




$$
\begin{aligned}
& =-\frac{2 z_{1} q^{\prime}\left(z_{1}\right)}{1+a^{2}} \\
& \geq k
\end{aligned}
$$

where $k \geq 1$ and $q\left(z_{1}\right)=i a$ and $a>0$. This shows that $z_{1} q^{\prime}\left(z_{1}\right)$ is a negative real number. This gives

$$
\mathfrak{I m} \frac{z_{1} q^{\prime}\left(z_{1}\right)}{q\left(z_{1}\right)}=\mathfrak{I m} \frac{z_{1} q^{\prime}\left(z_{1}\right)}{i a} \geq \frac{k}{2}\left(a+\frac{1}{a}\right) \geq k .
$$

On the other hand, we have

$$
\frac{z_{1} q^{\prime}\left(z_{1}\right)}{q\left(z_{1}\right)}=\frac{1}{\alpha} \frac{z_{1} p^{\prime}\left(z_{1}\right)}{p\left(z_{1}\right)}
$$

and putting $z=\left|z_{1}\right| e^{i \theta}$ in the section $\arg z_{1}-\varepsilon<\theta<\arg z_{1}+\varepsilon$, then $\arg \left\{q\left(z_{1}\right)\right\}$ takes its local maximum $\pi / 2$ at the point $\theta=\arg \left\{z_{1}\right\}$ in this section and so, we have

$$
\mathfrak{R e} \frac{z_{1} q^{\prime}\left(z_{1}\right)}{q\left(z_{1}\right)}=\left[\mathfrak{R e} \frac{\mathrm{d} \log q(z)}{\mathrm{d} z}\right]_{z=z_{1}}=\left[\frac{\mathrm{d} \arg q(z)}{\mathrm{d} \theta}\right]_{\theta=\arg z_{1}}=0 .
$$

Therefore, we have

$$
\frac{z_{1} p^{\prime}\left(z_{1}\right)}{p\left(z_{1}\right)}=i \frac{\alpha k}{2}\left(a+\frac{1}{a}\right), \quad \frac{\alpha k}{2}\left(a+\frac{1}{a}\right) \geq \alpha k
$$

where $p^{1 / \alpha}\left(z_{1}\right)=i a, a>0$. This completes the proof of (1.13).

For the next case arg $p\left(z_{2}\right)=-\pi \beta / 2$ let us put

$$
\rho(z)=[p(z)]^{1 / \beta}, \quad \psi(z)=\frac{1-\rho(z)}{1+\rho(z)},
$$

then it follows that

$$
\rho(0)=1, \rho^{\prime}(0)=\rho^{\prime \prime}(0)=\cdots=\rho^{(k-1)}(0)=0 \text { and } \rho^{(k)}(0) \neq 0
$$

and so

$$
\psi(0)=\psi^{\prime}(0)=\psi^{\prime \prime}(0)=\cdots=\psi^{(k-1)}(0)=0 \text { and } \psi^{(k)}(0) \neq 0
$$

From the hypothesis, we have

$$
\arg \rho(z)>-\frac{\pi}{2}, \quad z \in D_{\varepsilon}\left(z_{2}\right)=N_{\varepsilon}\left(z_{2}\right) \backslash\left\{z_{2}\right\}
$$

for some $\varepsilon>0$ and

$$
\arg \rho\left(z_{2}\right)=-\frac{\pi}{2} .
$$


Therefore, we have

$$
|\psi(z)|<1, \quad z \in D_{\varepsilon}\left(z_{2}\right)
$$

and

$$
\left|\psi\left(z_{2}\right)\right|=1 .
$$

This shows that $|\psi(z)|$ takes its local maximum value at the point $z=z_{2}$ in the the domain $N_{\varepsilon}\left(z_{2}\right)$ and applying Lemma 1.1, we have

$$
\begin{aligned}
\frac{z_{2} \psi^{\prime}\left(z_{2}\right)}{\psi\left(z_{2}\right)} & =-\frac{z_{2} \rho^{\prime}\left(z_{2}\right)}{1-\rho\left(z_{2}\right)}-\frac{z_{2} \rho^{\prime}\left(z_{2}\right)}{1+\rho\left(z_{2}\right)} \\
& =-\frac{2 z_{2} \rho^{\prime}\left(z_{2}\right)}{\left(1-\rho\left(z_{2}\right)\right)\left(1+\rho\left(z_{2}\right)\right)} \\
& =-\frac{2 z_{2} \rho^{\prime}\left(z_{2}\right)}{(1-i b)(1+i b)} \\
& =-\frac{2 z_{2} \rho^{\prime}\left(z_{2}\right)}{1+b^{2}} \\
& \geq k,
\end{aligned}
$$

where $k \geq 1$ and $\rho\left(z_{2}\right)=-i b$ with $b>0$. This shows that $z_{2} \rho^{\prime}\left(z_{2}\right)$ is a negative real number. This gives

$$
\mathfrak{I m} \frac{z_{2} \rho^{\prime}\left(z_{2}\right)}{\rho\left(z_{2}\right)}=\mathfrak{I m} \frac{z_{2} \rho^{\prime}\left(z_{2}\right)}{-i b} \leq \frac{k}{2}\left(b+\frac{1}{b}\right) \geq k .
$$

On the other hand, we have

$$
\frac{z_{2} \rho^{\prime}\left(z_{2}\right)}{\rho\left(z_{2}\right)}=\frac{1}{\beta} \frac{z_{2} p^{\prime}\left(z_{2}\right)}{p\left(z_{2}\right)}
$$

and putting $z=\left|z_{2}\right| e^{i \theta}$ in the section $\arg z_{2}-\varepsilon<\theta<\arg z_{2}+\varepsilon$, then $\arg \left\{\rho\left(z_{2}\right)\right\}$ takes its local minimum $-\pi / 2$ at the point $\theta=\arg \left\{z_{2}\right\}$ in this section and so, we have

$$
\mathfrak{R e} \frac{z_{2} \rho^{\prime}\left(z_{2}\right)}{\rho\left(z_{2}\right)}=\left[\mathfrak{R e} \frac{\mathrm{d} \log \rho(z)}{\mathrm{d} z}\right]_{z=z_{2}}=\left[\frac{\mathrm{d} \arg \rho(z)}{\mathrm{d} \theta}\right]_{\theta=\arg z_{2}}=0 .
$$

Therefore, we have

$$
\frac{z_{2} p^{\prime}\left(z_{2}\right)}{p\left(z_{2}\right)}=-i \frac{\beta k}{2}\left(b+\frac{1}{b}\right)
$$

is a pure imaginary number, where $p^{1 / \beta}\left(z_{2}\right)=-i b, b>0$ so

$$
-\frac{\beta k}{2}\left(b+\frac{1}{b}\right) \leq-\beta k
$$

This completes the proof of (1.14). 
Theorem 1.2 is a generalization of a result in [4,5].

Corollary 1.3 Under the assumptions of Theorem 1.2 we have

$$
\int_{z_{2}}^{z_{1}}\left\{\frac{z p^{\prime}(z)}{p(z)}\right\}^{\prime} \mathrm{d} z \geq(\alpha+\beta) k
$$

Corollary 1.3 implies that the length of image curve under $z p^{\prime}(z) / p(z)$ of the circular arc $|z|=\left|z_{1}\right|=\left|z_{2}\right|$ from $z_{2}$ to $z_{1}$ is great or equal to $(\alpha+\beta) k$.

Corollary 1.4 Under the assumptions of Theorem 1.2 and if $\arg z_{2}<\arg z_{1}$ and

$$
\left|\mathfrak{R e}\left[\left\{\frac{p^{\prime}(z)}{p(z)}+\frac{z p^{\prime \prime}(z)}{p^{\prime}(z)}-z\left(\frac{p^{\prime}(z)}{p(z)}\right)^{2}\right\} i z\right]\right|<\frac{1}{2 \pi}, \quad z \in \mathbb{D},
$$

then we have

$$
\mathfrak{R e}\{p(z)\}>0, \quad z \in \mathbb{D} .
$$

Proof From Lemma 1.1 we have

$$
0<\alpha+\beta \leq(\alpha+\beta) k \geq \alpha+\beta \leq\left|\int_{z_{2}}^{z_{1}}\left\{\frac{z p^{\prime}(z)}{p(z)}\right\}^{\prime} \mathrm{d} z\right|
$$

On the other hand and applying (1.15), we have

$$
\begin{aligned}
0<\alpha+\beta \leq\left|\int_{z_{2}}^{z_{1}}\left\{\frac{z p^{\prime}(z)}{p(z)}\right\}^{\prime} \mathrm{d} z\right| & =\left|\mathfrak{R e} \int_{\theta_{2}}^{\theta_{1}}\left\{\frac{p^{\prime}(z)}{p(z)}+\frac{z p^{\prime \prime}(z)}{p^{\prime}(z)}-z\left(\frac{p^{\prime}(z)}{p(z)}\right)^{2}\right\} i z \mathrm{~d} \theta\right| \\
& \leq \int_{\theta_{2}}^{\theta_{1}} \mid \mathfrak{R e}\left[\left\{\frac{p^{\prime}(z)}{p(z)}+\frac{z p^{\prime \prime}(z)}{p^{\prime}(z)}-z\left(\frac{p^{\prime}(z)}{p(z)}\right)^{2}\right\} i z\right] \mathrm{d} \theta \\
& \leq \frac{\theta_{1}-\theta_{2}}{2 \pi}<1,
\end{aligned}
$$

where $\arg z_{1}=\theta_{1}, \arg z_{2}=\theta_{2}$ and $\theta_{2}<\theta_{1}$. This shows that

$$
0<\alpha+\beta<1
$$

which implies $0<\alpha<1$ and $0<\beta<1$ or

$$
\mathfrak{R e}\{p(z)\}>0, \quad z \in \mathbb{D} \text {. }
$$

In $[8$, p.54] we can find the following result for the convolution of power series

$$
\sum_{n=0}^{\infty} a_{n} z^{n} * \sum_{n=0}^{\infty} b_{n} z^{n}=\sum_{n=0}^{\infty} a_{n} b_{n} z^{n}
$$


Lemma 1.5 If $g(z) \in \mathcal{C}, f(z) \in \mathcal{S}^{*}$ and $F(z)$ is analytic in $\mathbb{D}$, then for all $z \in \mathbb{D}$

$$
\frac{g(z) * F(z) f(z)}{g(z) * f(z)} \in \overline{c o} F(\mathbb{D}),
$$

where $\overline{c o} A$ denotes the closed convex hull of $A$.

Theorem 1.6 If $f(z)=a_{0}+a_{1} z+a_{2} z^{2}+\cdots, h(z)=b_{0}+b_{1} z+b_{2} z^{2}+\cdots \in \mathcal{H}$, $z h^{\prime \prime}(z) /\left(2 b_{2}\right) \in \mathcal{S}^{*}$ and

$$
q(z)=\frac{f^{\prime}(z)-a_{1}}{h^{\prime}(z)-b_{1}}, \quad p(z)=\frac{f^{\prime \prime}(z)}{h^{\prime \prime}(z)}, \quad z \in \mathbb{D},
$$

are analytic functions in $\mathbb{D}$, then

$$
q(\mathbb{D}) \subseteq \overline{c o} p(\mathbb{D}) .
$$

Proof Denote

$$
s(z)=\sum_{n=2}^{\infty} \frac{z^{n-1}}{n-1}=-\log (1-z), \quad z \in \mathbb{D},
$$

then $s(z) \in \mathcal{C}$.

Applying Lemma 1.5, we have

$$
\begin{aligned}
\frac{f^{\prime}(z)-a_{1}}{h^{\prime}(z)-b_{1}} & =\frac{\sum_{n=2}^{\infty} n a_{n} z^{n-1}}{\sum_{n=2}^{\infty} n b_{n} z^{n-1}} \\
& =\frac{\sum_{n=2}^{\infty} n a_{n} z^{n-2}}{\sum_{n=2}^{\infty} n b_{n} z^{n-2}} \\
& =\frac{\sum_{n=2}^{\infty} \frac{z^{n-2}}{n-1} * \sum_{n=2}^{\infty} n(n-1) a_{n} z^{n-2}}{\sum_{n=2}^{\infty} \frac{z^{n-2}}{n-1} * \sum_{n=2}^{\infty} n(n-1) b_{n} z^{n-2}} \\
& =\frac{\frac{s(z)}{z} * f^{\prime \prime}(z)}{\frac{s(z)}{z} * h^{\prime \prime}(z)} \\
& =\frac{\frac{s(z)}{z} * p(z) h^{\prime \prime}(z)}{\frac{s(z)}{z} * h^{\prime \prime}(z)} \\
& =\frac{s(z) * p(z) \frac{z h^{\prime \prime}(z)}{2 b_{2}}}{s(z) * \frac{z h^{\prime \prime}(z)}{2 b_{2}}} \\
& \in \frac{\operatorname{co} p(\mathbb{D})}{}
\end{aligned}
$$

because $s(z) \in \mathcal{C}$ and $z h^{\prime \prime}(z) /\left(2 b_{2}\right) \in \mathcal{S}^{*}$.

The differential subordination theory provides another method od proving this type of results. For two functions $f(z)$ and $g(z)$ analytic in $\mathbb{D}$, we say that $f f(z)$ is subor- 
dinate to $g(z)$, written by $f(z) \prec g(z)$, if there exists a function $w(z)$, analytic in $\mathbb{D}$, with $w(0)=0$ and $|w(z)|<1$ such that $f(z)=g(w(z))$.

In $[3$, p.70] we can find the following result

Lemma 1.7 Let $H(z)$ be convex univalent, $P(z) \in \mathcal{H}$ and $\mathfrak{R e}\{P(z)\}>0$ in $\mathbb{D}$. If $p(z)$ is amalytic in $\mathbb{D}$ with $p(0)=H(0)$, then

$$
p(z)+P(z) z p^{\prime}(z) \prec H(z) \quad \Rightarrow \quad p(z) \prec H(z) .
$$

Theorem 1.8 Let $H(z)$ be convex univalent in $\mathbb{D}$. Assume that

$$
\frac{f^{(s)}(z)}{g^{(s)}(z)}, \quad \frac{f^{(s-1)}(z)}{g^{(s-1)}(z)}, \quad \frac{g^{(s-1)}(z)}{z g^{(s)}(z)}
$$

are analytic in $\mathbb{D}$ for some positive integer $s$ and

$$
\mathfrak{R e}\left\{\frac{g^{(s-1)}(z)}{z g^{(s)}(z)}\right\}>0, \quad z \in \mathbb{D} .
$$

If

$$
\frac{f^{(s)}(z)}{g^{(s)}(z)} \prec H(z),
$$

and with $f^{(s-1)}(0) / g^{(s-1)}(0)=H(0)$ then

$$
\frac{f^{(s-1)}(z)}{g^{(s-1)}(z)} \prec H(z) .
$$

Proof Denote

$$
p(z)=\frac{f^{(s-1)}(z)}{h^{(s-1)}(z)} .
$$

Then we have $p(z) h^{(s-1)}(z)=f^{(s-1)}(z)$, by differentation, we obtain

$$
p(z)+\frac{h^{(s-1)}(z)}{z h^{(s)}(z)} z p^{\prime}(z)=\frac{f^{(s)}(z)}{h^{(s)}(z)} \prec H(z) .
$$

Applying (1.17) and (1.18) give $p(z) \prec H(z)$, i. e. (1.19).

Corollary 1.9 Assume that $\alpha \in(0,1]$ and

$$
\frac{f^{\prime \prime}(z)}{h^{\prime \prime}(z)}, \quad \frac{f^{\prime}(z)}{h^{\prime}(z)}, \quad \frac{h(z)}{z h^{\prime}(z)}, \frac{h^{\prime}(z)}{z h^{\prime \prime}(z)}
$$

are analytic in $\mathbb{D}$ Then

$$
\frac{f^{\prime}(z)}{h^{\prime}(z)} \prec\left\{\frac{1+z}{1-z}\right\}^{\alpha} \text { and } \mathfrak{R e}\left\{\frac{h(z)}{z h^{\prime}(z)}\right\}>0, \quad z \in \mathbb{D}, \Rightarrow \frac{f(z)}{h(z)} \prec\left\{\frac{1+z}{1-z}\right\}^{\alpha}
$$


and

$$
\frac{f^{\prime \prime}(z)}{h^{\prime \prime}(z)} \prec\left\{\frac{1+z}{1-z}\right\}^{\alpha} \text { and } \mathfrak{R e}\left\{\frac{h^{\prime}(z)}{z h^{\prime \prime}(z)}\right\}>0, \quad z \in \mathbb{D}, \quad \Rightarrow \frac{f^{\prime}(z)}{h^{\prime}(z)} \prec\left\{\frac{1+z}{1-z}\right\}^{\alpha} .
$$

Consider, $h(z)=e^{z}-1$, which satisfies

$$
\mathfrak{R e} \frac{z h^{\prime \prime}(z)}{h^{\prime}(z)}=\mathfrak{R e}\{z\}>-1, \quad z \in \mathbb{D} .
$$

Therefore, $h(z) \in \mathcal{C}$ and so $h(z) \in \mathcal{S}^{*}$ which gives

$$
\mathfrak{R e}\left\{\frac{h(z)}{z h^{\prime}(z)}\right\}>0, \quad z \in \mathbb{D} .
$$

Applying (1.20) we obtain

$$
\frac{f^{\prime}(z)}{e^{z}} \prec\left\{\frac{1+z}{1-z}\right\}^{\alpha} \Rightarrow \frac{f(z)}{e^{z}-1} \prec\left\{\frac{1+z}{1-z}\right\}^{\alpha} .
$$

Corollary 1.10 Assume that $\alpha \in[0,1)$ and

$$
\frac{f^{\prime \prime}(z)}{h^{\prime \prime}(z)}, \quad \frac{f^{\prime}(z)}{h^{\prime}(z)}, \quad \frac{h(z)}{z h^{\prime}(z)}, \frac{h^{\prime}(z)}{z h^{\prime \prime}(z)}
$$

are analytic in $\mathbb{D}$. Then

$$
\mathfrak{R e}\left\{\frac{h(z)}{z h^{\prime}(z)}\right\}>0 \text { and } \mathfrak{R e} \frac{f^{\prime}(z)}{h^{\prime}(z)}>\alpha, \quad z \in \mathbb{D} \Rightarrow \mathfrak{R e} \frac{f(z)}{h(z)}>\alpha, \quad z \in \mathbb{D}
$$

and

$$
\mathfrak{R e}\left\{\frac{h^{\prime}(z)}{z h^{\prime \prime}(z)}\right\}>0 \text { and } \mathfrak{R e} \frac{f^{\prime \prime}(z)}{h^{\prime \prime}(z)}>\alpha, \quad z \in \mathbb{D} \Rightarrow \mathfrak{R e} \frac{f^{\prime}(z)}{h^{\prime}(z)}>\alpha, \quad z \in \mathbb{D} \text {. }
$$

Property (1.22) was known earlier, see Theorem $2.6 f$ in [3, p.63].

Theorem 1.11 Assume that $h(z) \in \mathcal{H}$ and

$$
\mathfrak{R e} \frac{h^{\prime}(z)-\gamma-z}{z\left(h^{\prime \prime}(z)-1\right)}>0, \quad z \in \mathbb{D}
$$

for some $\gamma \in(1, \infty]$ and

$$
\left|\arg \left\{h^{\prime}(z)-\gamma-z\right\}\right|<\frac{\beta \pi}{2}, \quad z \in \mathbb{D},
$$


for some $\beta \in(0,1]$. Assume that $f(z) \in \mathcal{H}$ and

$$
\left|\arg \frac{f^{\prime \prime}(z)-1}{h^{\prime \prime}(z)-1}\right|<\frac{\alpha \pi}{2}, \quad z \in \mathbb{D},
$$

for some $\alpha \in(0,1]$. Furthermore, assume that

$$
F(z)=\frac{f^{\prime}(z)-\gamma-z}{h^{\prime}(z)-\gamma-z}
$$

is analytic in $\mathbb{D}$ with $F(0)=1$. Then

$$
\left|\arg \left\{f^{\prime}(z)\right\}\right|<\frac{(\alpha+\beta) \pi}{2}, \quad z \in \mathbb{D} .
$$

Proof Consider Theorem 1.8 with $s=2, H(z)=\left(\frac{1+z}{1-z}\right)^{\alpha}$ and $g^{\prime}(z)=h^{\prime}(z)-\gamma-z$. Then $H(z)$ is convex univalent and condition (1.18) becomes (1.24). Applying this for (1.26) gives us

$$
\left|\arg \left\{\frac{f^{\prime}(z)-\gamma-z}{h^{\prime}(z)-\gamma-z}\right\}\right|<\frac{\alpha \pi}{2}, \quad z \in \mathbb{D} .
$$

Now, applying (1.25), we have

$$
\begin{aligned}
& \left|\arg \left\{f^{\prime}(z)-\gamma-z\right\}\right|-\left|\arg \left\{h^{\prime}(z)-\gamma-z\right\}\right| \\
\leq & \left|\arg \left\{f^{\prime}(z)-\gamma-z\right\}-\arg \left\{h^{\prime}(z)-\gamma-z\right\}\right| \\
= & \left|\arg \left\{\frac{f^{\prime}(z)-\gamma-z}{h^{\prime}(z)-\gamma-z}\right\}\right| \\
< & \frac{\alpha \pi}{2}, \quad z \in \mathbb{D}
\end{aligned}
$$

because of (1.28). So we have

$$
\begin{aligned}
\left|\arg \left\{f^{\prime}(z)-\gamma-z\right\}\right| & \leq \frac{\alpha \pi}{2}+\left|\arg \left\{h^{\prime}(z)-\gamma-z\right\}\right| \\
& <\frac{(\alpha+\beta) \pi}{2}, \quad z \in \mathbb{D} .
\end{aligned}
$$

A simple geometric observation shows that

$$
\left|\arg \left\{f^{\prime}(z)\right\}\right|<\left|\arg \left\{f^{\prime}(z)-\gamma-z\right\}\right|, \quad z \in \mathbb{D}, \quad \gamma \in(1, \infty] .
$$

Therefore,

$$
\left|\arg \left\{f^{\prime}(z)\right\}\right|<\left|\arg \left\{f^{\prime}(z)-\gamma-z\right\}\right|<\frac{(\alpha+\beta) \pi}{2}, \quad z \in \mathbb{D}
$$


Condition (1.27) implies that $f(z)$ is strongly close-to-convex of order $\alpha+\beta$ with respect convex function $g(z)=z$. We obtain more when $\alpha+\beta \leq 1$ in the following corollary.

Corollary 1.12 Assume that $h(z) \in \mathcal{H}$ and

$$
\mathfrak{R e} \frac{h^{\prime}(z)-\gamma-z}{z\left(h^{\prime \prime}(z)-1\right)}>0, \quad z \in \mathbb{D}
$$

for some $\gamma \in(1, \infty]$ and

$$
\left|\arg \left\{h^{\prime}(z)-\gamma-z\right\}\right|<\frac{\beta \pi}{2}, \quad z \in \mathbb{D}
$$

for some $\beta \in(0,1]$. Assume that $f(z) \in \mathcal{H}$ and

$$
\left|\arg \frac{f^{\prime \prime}(z)-1}{h^{\prime \prime}(z)-1}\right|<\frac{\alpha \pi}{2}, \quad z \in \mathbb{D},
$$

for some $\alpha \in(0,1-\beta]$. Furthermore, assume that

$$
F(z)=\frac{f^{\prime}(z)-\gamma-z}{h^{\prime}(z)-\gamma-z}
$$

is analytic in $\mathbb{D}$ with $F(0)=1$. Then

$$
\mathfrak{R e}\left\{f^{\prime}(z)\right\}>0, \quad z \in \mathbb{D} .
$$

Proof We have $\alpha+\beta \leq 1$, then by Theorem 1.11, we have

$$
\left|\arg \left\{f^{\prime}(z)\right\}\right|<\frac{(\alpha+\beta) \pi}{2} \leq \frac{\pi}{2}, \quad z \in \mathbb{D} .
$$

This gives (1.32).

Condition (1.32) implies the univelence $f(z)$ in $\mathbb{D}$ by Noshiro-Warshawski's theorem.

Data Availability The datasets generated during and/or analysed during the current study are available from the corresponding author on reasonable request.

\section{Compliance with ethical standards}

Conflict of interest The author declares no conflict of interest. 
Open Access This article is licensed under a Creative Commons Attribution 4.0 International License, which permits use, sharing, adaptation, distribution and reproduction in any medium or format, as long as you give appropriate credit to the original author(s) and the source, provide a link to the Creative Commons licence, and indicate if changes were made. The images or other third party material in this article are included in the article's Creative Commons licence, unless indicated otherwise in a credit line to the material. If material is not included in the article's Creative Commons licence and your intended use is not permitted by statutory regulation or exceeds the permitted use, you will need to obtain permission directly from the copyright holder. To view a copy of this licence, visit http://creativecommons.org/licenses/by/4.0/.

\section{References}

1. Fukui, S., Sakaguchi, K.: An extension of theorem of Ruscheweyh. Bull. Fac. Educ. Wakayama Univ. Nat. Sci. 29, 1-3 (1980)

2. Jack, I.S.: Functions starlike and convex of order $\alpha$. J. Lond. Math. Soc. 3, 469-474 (1971)

3. Miller, S.S., Mocanu, P.T.: Differential Subordinations, Theory and Applications, Series of Monographs and Textbooks in Pure and Applied Mathematics, vol. 225. Marcel Dekker Inc., New York (2000)

4. Nunokawa, M.: On properties of non-Carathéodory functions. Proc. Jpn. Acad. Ser. A 68(6), 152-153 (1992)

5. Nunokawa, M.: On the order of strongly starlikeness of strongly convex functions. Proc. Jpn. Acad. Ser. A 69(7), 234-237 (1992)

6. Robinson, R.M.: Univalent majorants. Trans. Am. Math. Soc. 61, 1-35 (1947)

7. Pommerenke, Ch.: On close-to-convex analytic functions. Trans. Am. Math. Soc. 114(1), 176-186 (1964)

8. Ruscheweyh, St.: Convolution in geometric function theory, Les Presses de l'Université de Montréal (1982)

Publisher's Note Springer Nature remains neutral with regard to jurisdictional claims in published maps and institutional affiliations. 